\title{
Interactions between $A P O E$ genotype and plasma fatty acids on cardiometabolic risk markers in individuals with the Metabolic Syndrome
}

\author{
R. Fallaize ${ }^{1}$, A.L. Carvalho-Wells ${ }^{1}$, K. Ayres ${ }^{2}$, A. Dembinska-Kiec ${ }^{3}$, C.A. Drevon ${ }^{4}$, \\ C. DeFoort ${ }^{5}$, J. Lopez-Miranda ${ }^{6}$, U. Riserus ${ }^{7}$, E. Blaak ${ }^{8}$, H.M. Roche ${ }^{9}$ and J.A. Lovegrove ${ }^{1}$ \\ ${ }^{1}$ Hugh Sinclair Unit of Human Nutrition and Institute for Cardiovascular and Metabolic Research, University of \\ Reading, RG6 6AP, UK, ${ }^{2}$ Department of Mathematics and Statistics, University of Reading, RG6 6AX, UK, \\ ${ }^{3}$ Department of Clinical Biochemistry, University Medical College, Krakow, Poland, ${ }^{4}$ Department of Clinical \\ Endocrinology, Oslo University Hospital Oslo, Norway, ${ }^{5}$ INSERM, 476 Human Nutrition and Lipids, Marseille, \\ France, ${ }^{6}$ Lipid and Atherosclerosis Unit, IMIBIC/Reina Sofia University Hospital/University of Cordoba, Spain, \\ ${ }^{7}$ Department of Public Health and Caring Sciences/Clinical Nutrition and Metabolism, Uppsala University, Uppsala, \\ Sweden, ${ }^{8}$ NUTRIM School for Nutrition and Translational Research in Metabolism, Maastricht University Medical \\ Centre + (MUMC+) Maastricht, The Netherlands and ${ }^{9}$ Nutrigenomics Research Group, UCD Conway Institute, \\ Dublin, Ireland
}

The $\varepsilon 4$ allele of the $A P O E$ gene has been associated with higher TC, LDL-C and risk of cardiovascular disease (CVD) ${ }^{(1)}$, and increased responsiveness to dietary saturated fat and cholesterol $^{(2)}$. Given that individuals with the Metabolic Syndrome (MetS) have a four-fold increased risk of $\mathrm{CVD}^{(3)}$, they are an ideal target for gene-based nutrition interventions. However, the extent to which MetS traits are affected by interactions between the $A P O E$ genotype and plasma fatty acids (FA) is unknown.

The aim of the present analysis was to explore nutrient-gene interactions between the $A P O E$ polymorphism and plasma FA concentrations on metabolic markers in individuals with the MetS. To achieve this, plasma FA, blood pressure, insulin sensitivity, lipid concentrations and APOE genotype were determined in a cross-sectional analysis of 442 MetS individuals who participated in the LIPGENE study. Adjusted general linear models were used to assess nutrient-gene interactions at baseline.

A geographic cline was observed with respect to $\varepsilon 4$ allele frequency, with $22.8 \%$ frequency observed in Norway compared with $8.6 \%$ in Spain. E4 carriers had higher plasma concentrations of TC $(P=0.004)$, LDL-C $(P<0.001)$, and apo B $(P<0.001)$ compared with the $E 2$ carriers; and lower TC $(P=0.013)$, LDL-C $(P=0.004)$ and apoB $(P=<0.001)$ compared to the E3/E3 group. High plasma n-3 PUFA was associated with a lower concentration of apoCIII in $E 2$ carriers $(P=0.020)$. High plasma $\mathrm{C} 16: 0$ was associated with insulin resistance (HOMA-IR) in $E 4$ carriers $(P=0 \cdot 001)$.

A detrimental impact of plasma SFA on insulin resistance was observed in $E 4$ carriers with MetS. In $E 2$ carriers, higher n-3 PUFA was associated with lower apoCIII concentrations. These findings suggest that individuals with MetS might benefit from personalised dietary advice targeted to $A P O E$ genotype, although further confirmatory intervention studies are required. This trial was registered at clinicaltrials.gov as NCT00429195.

1. Bennet AM, Di Angelantonio E, Ye Z et al. (2007) JAMA 298(11), 1300-1311

2. Masson LF, McNeill G \& Avenell A (2003) Am J Clin Nutr 77(5), 1098-1111

3. Lau DC. (2009) Curr Atheroscler Rep 11(4), 264-271 